INPLASY

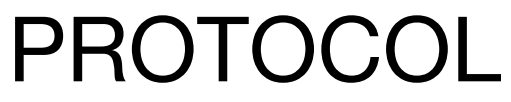

To cite: Guo et al. Vitamin D supplementation and PCOS: A systematic review of RCTs and insight into the underlying mechanism. Inplasy protocol 202040010. doi:

10.37766/inplasy2020.4.0010

Received: 03 April 2020

Published: 03 April 2020

Corresponding author: Ying Liu

yingliubj@hotmail.com

Author Affiliation:

Capital Medical University

Support: National Natural Science Found

Review Stage at time of this submission: Data analysis.

Conflicts of interest:

The authors declare no conflict of interest.

\section{Vitamin D supplementation and PCOS: A systematic review of RCTs and insight into the underlying mechanism}

Guo, S1; Liu, Y2.

Review question / Objective: Patients: polycystic ovary syndrome(PCOS) women; Intervention: vitamin D supplementation alone; Control: placebo; Outcome:vitamin D level, FPG, insulin, HOMA-IR, QUICKI, triglycerides, total-C, LDL-C, HDL-C, VLDL-C, total-T, DHEAS, SHBG, hs-CRP; Study Design: meta-analysis.

Rationale: Most of published meta-analysis on this aspect included either non-RCTs or co-supplemented trials (with metformin or other nutrients), which might mask the real impact of vitamin D supplementation. Moreover, those published reviews tended to focuse on one specific area, which might prevent us to getting thorough understanding of the potential effect of vitamin D supplementation. Thus, we conducted a comprehensive systematic review and meta-analysis to get a more convinced conclusion.

INPLASY registration number: This protocol was registered with the International Platform of Registered Systematic Review and Meta-Analysis Protocols (INPLASY) on 03 April 2020 and was last updated on 03 April 2020 (registration number INPLASY202040010.

\section{INTRODUCTION}

Review question / Objectives: Patients: polycystic ovary syndrome(PCOS) women; Intervention: vitamin D supplementation alone; Control: placebo; Outcome:vitamin D level, FPG, insulin, HOMA-IR, QUICKI, triglycerides, total-C, LDL-C, HDL-C, VLDL-
C, total-T, DHEAS, SHBG, hs-CRP; Study Design: meta-analysis.

Condition being studied: PCOS.

Rationale: Most of published meta-analysis on this aspect included either non-RCTs or co-supplemented trials (with metformin or 
other nutrients), which might mask the real impact of vitamin D supplementation. Moreover, those published reviews tended to focuse on one specific area, which might prevent us to getting thorough understanding of the potential effect of vitamin D supplementation. Thus, we conducted a comprehensive systematic review and meta-analysis to get a more convinced conclusion.

\section{METHODS}

Participant or population: PCOS patients.

Intervention: vitamin D supplementation alone.

Comparator: placebo.

Study designs to be included: Randomized controlled trials (RCTS).

Eligibility criteria: 1) RCTs; 2) study subjects were women diagnosed with PCOS; 3) PCOS was diagnosed on the basis of the 2003 Rotterdam criteria or the 1990 National Institute of Child Health and Human Development criteria; 4) full text was accessible; 5) the study compared the therapeutic effect of vitamin D supplement with placebo.

Information sources: Pubmed, Embase, Web of Science, Cochrane Library and Clinical Trails. Ifrequired information was incomplete, authors were contacted.

Main outcome(s): Serum vitamin D level, fasting plasma glucose (FPG), fasting insulin, HOMA-IR, QUICKI, serum triglyceride (TG), total cholesterol (TC), high-density lipoprotein cholesterol (HDLC), low-density lipoprotein cholesterol (LDL-C), very low-density lipoprotein cholesterol (VLDL-C), hypersensitive Creactive protein (hs-CRP), total testosterone (Total T), sex hormone-binding globulin (SHBG); dehydroepiandrosterone sulfate (DHEAS) were collected after whole course of vitamin $D$ supplementation.

Quality assessment / Risk of bias analysis: The cochrane risk of bias assessment tool was deployed to evaluate the method of randomization, allocation of concealment, blinding of participants, personnel and outcome assessment, incomplete outcome, selective reporting and other bias. Risk bias of each study was graded as low, unclear and high.

Strategy of data synthesis: Statistical synthesis and subgroup analysis were performed by Revman V.5.3 offered by the Cochrane Collaboration. Measurement data were displayed as mean difference and standard deviation. Mean difference (MD) with $95 \%$ confidence intervals (CI) by inverse variance method was employed in case of data with identical measuring units otherwise Standard mean difference (SMD) was adopted. Heterogeneity among studies was estimated by Cochran's $Q$ test and Isquared. $\mathrm{P} 50 \%$ indicates statistical heterogeneity. When significant heterogeneity presented, the randomeffects model of meta-analysis was applied.

Search strategy: Pubmed, Embase, Web of Science, Cochrane Library and Clinical Trails were searched up to March 2020 with the following MeSH and non-MeSH terms: vitamin D, vitamin D3, vitamin D2, cholecalciferol, ergocalciferols, hydroxycholecalciferol or calcitriol combined with polycystic ovary syndrome, ovary polycystic disease, PCOS, steinLeventhal syndrome, stein Leventhal syndrome or polycystic ovary syndrome. The reference lists of related literatures were also browsed to identify any potential additional publications. No restrictions were made for language or date of publications.

Subgroup analysis: 1) vitamin D supplemantation interval: day, week, two w e e k s, 20 da ys; 2 ) vit a m in D supplemantation dose: low dose $(50,000 / \mathrm{J} /$ week), high dose; 3)serum vitamin D level of PCOS patients: vitamin D insufficient group, vitamin D deficient group.

Sensibility analysis: Sensibility analysis was done by change of statistical method (MD 
change to SMD) and removal of each concluded study.

Language: Without language restrictions.

Coutries involved: Iran, India, Austria, Venezuela and the United States.

Keywords: vitamin D; PCOS; meta-analysis. 